\title{
Surface-Engineered Cubosomes Serve as a Novel Vaccine Adjuvant to Modulate Innate Immunity and Improve Adaptive Immunity in vivo
}

This article was published in the following Dove Press journal: International Journal of Nanomedicine

\author{
Zhenguang Liu ${ }^{1,2}$ \\ Lin $\mathrm{Yu}^{1,2}$ \\ Pengfei Gu ${ }^{1,2}$ \\ Ruonan Bol,2 \\ Shuwen $\mathrm{Xu}^{1,2}$ \\ Adelijiang Wusiman ${ }^{1,2}$ \\ Jiaguo Liu $\mathbb{D}^{1,2}$ \\ Yuanliang $\mathrm{Hu}^{1,2}$ \\ Deyun Wang (D) ${ }^{1,2}$
}

\begin{abstract}
'Institute of Traditional Chinese Veterinary Medicine, College of Veterinary Medicine, Nanjing Agricultural University, Nanjing 210095, People's Republic of China; ${ }^{2}$ MOE Joint International Research Laboratory of Animal Health and Food Safety, College of Veterinary Medicine, Nanjing Agricultural University, Nanjing 210095, People's Republic of China
\end{abstract}

Objective: Recent studies have revealed the adjuvant activity of cubosomes and their potential utility as an antigen delivery system. In this study, to further enhance the adjuvant activity of cubosomes, two cationic polymers are modified on the surface of cubosomes.

Methods: Here, we exploit the effects of surface chemistry on the adjuvant activity of Ganoderma lucidum polysaccharide cubosomes (GLPC) by placing two kinds of molecules, that is, cetyltrimethylammonium bromide (CTAB) and poly(diallydimethyl ammonium chloride) (PDDAC), on their surface.

Results: CTAB- or PDDAC-modified GLPC were found to significantly promote humoral and cellular immune responses, as well as the proliferation of CD3+ CD4+ or $\mathrm{CD} 3+\mathrm{CD} 8+\mathrm{T}$ cells through the powerful activation of dendritic cells (DCs). The enhanced immune responses of PDDAC-modified GLPC might be attributed to the maturation of DCs into draining lymph nodes and the activation of spleen and cytokines in serum.

Conclusion: PDDAC modification is beneficial for enhancing humoral and cellular immune response, suggesting that PDDAC-GLPC-OVA has the ability to be a potential adjuvant for vaccine.

Keywords: cubosomes, Ganoderma lucidum polysaccharide, surface-engineering, immune response, RNA-seq

\section{Introduction}

Vaccination is an essential way for protecting the body from bacterial or virus infection and cancer. An effective vaccine should motivate strong humoral immune responses and cellular immune responses. The success of vaccine mainly includes antigen and matching adjuvants, which were necessary for playing the role of protecting the body.

Food and Drug Administration has approved four adjuvants (aluminum salts, MF59, AS03, and AS04), which were applied on a variety of bacteria, virus, and influenza vaccine. ${ }^{1-3}$ With the development of novel antigen (such as subunit antigen) technology, safer and more effective adjuvant are required. In recent years, nanotechnology and nanomaterial has acquired remarkable progress and been applied on biology and medicine. ${ }^{4,5}$ So far, a variety of nanomaterials have been developed into adjuvants, for example, liposome, PLGA, and cubosomes. ${ }^{6-9}$ Among these, the adjuvant function of cubosomes was discovered in 2013. The
Correspondence: Deyun Wang Institute of Traditional Chinese Veterinary Medicine, College of Veterinary Medicine Nanjing Agricultural University, Nanjing 210095 , People's Republic of China

Tel +86-25-84395203

Fax +86-25-84398669

Email dywang@njau.edu.cn 
researchers found cubosomes have the ability to promote more humoral and cellular immune responses than antigen alone. $^{10,11}$

The fungal species Ganoderma lucidum has been widely used as a traditional Chinese medicine for more than 2000 years. G. lucidum polysaccharide (GLP), one of the vital components of G. lucidum, plays a crucial role in the biological effects of the fungus. Their structures and pharmacological effects have been extensively studied. ${ }^{12}$ Modern research shows that GLP has excellent immunomodulating activities and has the ability to be a potential adjuvant for vaccine. ${ }^{13}$ However, GLP has no ability to target antigen presenting cells, which limits its extensive application as a vaccine adjuvant.

Cubosomes show colloidal dispersions of the bicontinuous cubic liquid crystalline phase and have a larger surface area than spherical nanoparticles, which confers them with a powerful ability of encapsulating drug or antigen. ${ }^{14-18}$ However, their immunogenicity is influenced by their physicochemical properties. Surface engineering of nanoparticles plays a critical role in modification of their physicochemical properties, enhancing vaccine-triggered immune responses and resulting in robust and lasting immune response when applied on subunit vaccine. ${ }^{19,20}$ Recent research has indicated that coating cubosomes with a single layer of poly- $\varepsilon$-lysine on enhances their functionality and anticancer properties. ${ }^{21}$ Accordingly, the linking of chemical molecules directly or indirectly on the surface of various nanoparticles has been shown to effectively induce the activation of DCs and enhance both humoral and cellular immune responses. $^{22-24}$

Our previous studies have shown that cubosomes enhance the targeting and adjuvant activity of GLP. ${ }^{25}$ However, GLP-cubosomes nanoparticles had shown poorly effects on the carry of subunit antigen and activation of APCs. In this study, we linked cetyltrimethylammonium bromide (CTAB) and poly (diallydimethy ammoniumchloride) (PDDAC) directly or indirectly on the surface of Ganoderma lucidum polysaccharide cubosomes (GLPC). We hypothesized that CTAB-GLPC and PDDAC-GLPC nanoparticles would function as an effective adjuvant and vaccine system to target and activate the APCs, and induce strong humoral and cell-mediated immune responses. This hypothesis was tested by investigating the effects of CTAB-GLPC and PDDAC-GLPC nanoparticles on the activation of DCs. Furthermore, ovalbumin (OVA) antigens were adsorbed on CTAB-GLPC and PDDAC-GLPC nanoparticles and their ability to induce humoral and cell-mediated immune responses was investigated. Flow cytometry and RNA-seq were used to measure the activation of DCs in draining lymph nodes and gene expression of the spleen to clarify its adjuvant mechanism.

\section{Materials and Methods \\ Materials}

Phytantriol (98.0\%) was obtained from TCI (Tokyo, Japan). Pluronic127 (F127), cetyltrimethylammonium bromide (CTAB), poly (styrene sulfate) (PSS, molecular weight: 70,000) and poly (diallydimethy ammoniumchloride (PDDAC, Mw=100,000-200,000)) were purchased from Sigma-Aldrich Co (St Louis, MO, USA). The purified G. lucidum polysaccharide ( $\geq 98 \%$ purity) was purchased from CiYuan Biotechnology Co, Ltd, Shanxi, People's Republic of China. Propylene glycol was purchased from Yuwang Industrial Co, Ltd (Shandong, People's Republic of China). 3-(4,5-Dimethylthiazol2-yl)-2,5-diphenyltetrazolium bromide (MTT) was purchased from Amresco LLC (Solon, OH, USA). RPMI 1640 was purchased from ThermoFisher Scientific (Waltham, MA, USA).

\section{Characterization of GLP}

The carbohydrate content of GLP was determined using the phenol sulfuric acid method. The homogeneity and molecular weight were determined by gel permeation chromatography (GPC). Man, Rha, GlcA, GalA, Glc, Gal, Ara, and Fuc were used as the monosaccharide standards, and the monosaccharide composition was analyzed by High Performance Liquid Chromatography (HPLC).

\section{Synthesis of GLPC and CTAB-GLPC}

The preparation of GLPC was assessed as described previously. ${ }^{25}$ CTAB-GLPC was prepared by adding CTAB (3 mg) to Phytantriol (200 mg) - Ethanol mixture, the subsequent method was used as per the previous description.

\section{Synthesis of PDDAC-GLPC}

PDDAC-GLPC was synthesized by layer-by-layer assembly. First, to prepare negatively charged cubosomes, PSS $(2 \%)$ solution were added into the aqueous solution of CTAB-GLPC and stirred for 15 minutes at $25^{\circ} \mathrm{C}$. The mixture sample was stood for 2 hours at $25^{\circ} \mathrm{C}$. Redundant PSS 
was removed by a filter membrane and CTAB-GLPC-PSS was obtained. For further synthesis of PDDAC-GLPC, similar steps were applied to coat with PDDAC (2.5\%) on the surface of CTAB-GLPC-PSS. Redundant PDDAC was removed by a filter membrane.

\section{Characterization of Cubosomes-Based Nanoparticles}

Zeta potential, sizes, and polydispersity index (PDI) of various cubosomes-based nanoparticles were measured using the Zetasizer Nano ZS90 instrument (Malvern Instruments Co., Ltd.). Small-angle X-ray scattering (SAXS) of various cubosomes-based nanoparticles were performed by a Bruker NanoSTAR SAXS. After equilibration at $25^{\circ} \mathrm{C}$ for 30 minutes, measurement was performed and a scattering pattern was collected for 30 minutes. Cryo-field emission scanning electron microscopy (CryoFESEM) was used to measure the surface and morphology of cubosomes-based nanoparticles. The various cubosomes-based nanoparticles were viewed using a microscope containing cryochamber at $-140^{\circ} \mathrm{C}$ with an accelerating voltage of $3 \mathrm{kV}$.

\section{The Effects of Cubosomes-Based Nanoparticles on the Maturation of Dendritic Cells (DCs)}

DCs from the bone marrow of BALB/c mice were collected and cultured according to the previous described method. ${ }^{23}$ All animal handling procedures were performed according to the Guide for the Care and Use of Laboratory Animals of the National Institutes of Health and followed the guidelines of the Animal Welfare Act. The Institutional Animal Care and Use Committee (IACUC) of Nanjing Agricultural University approved all animal procedures (Approval ID: 2011BAD34B02). Briefly, the bone marrow of the femur and tibia were collected and red blood cells were removed. Then, $3 \mathrm{~mL}$ cells were added into 6 -well plates at $1 \times 10^{6}$ per well, which contain $20 \mathrm{ng} / \mathrm{mL}$ GM-CSF (Peprotech/Tebu, Germany) and $20 \mathrm{ng} / \mathrm{mL} \quad$ IL-4 (Peprotech/Tebu). On days 2 and 4, all medium was removed and $3 \mathrm{~mL}$ of fresh medium was added to the cell well. On day $6,1.5 \mathrm{~mL}$ medium was removed and $1.5 \mathrm{~mL}$ of fresh medium was added to the cell well. On day 8, DCs were obtained and ready for subsequent experiment. DCs were treated with GLP, Cub, GLPC, CTAB-GLPC, or PDDAC-GLPC, respectively, for 12 hours. The concentrations of various cubosomes-based nanoparticles were fixed at $10 \mu \mathrm{g} / \mathrm{mL}$ (phytantriol content), while that of GLP was $1 \mu \mathrm{g} / \mathrm{mL}$. Lipopolysaccharide (LPS, Sigma) at $5 \mu \mathrm{g} / \mathrm{mL}$ was used as the positive control while untreated DCs was used as the negative control. DCs were collected and stained with antiCD11c-PE-Cy7, anti-CD86-FITC, and anti-CD80-PE antibodies (eBioscience) for 30 minutes at $4^{\circ} \mathrm{C}$. After removing redundant antibodies by washing, cells were analyzed for DCs mature.

\section{Immunization Protocol}

Female BALB/c mice (6-8 weeks old) were injected subcutaneously with $0.2 \mathrm{~mL}$ of either OVA alone or various cubosome-based nanoparticles/OVA complex. The various cubosomes-based nanoparticles/OVA complex was prepared by simple mixing and then incubated for 30 minutes. The concentrations of different components in various cubosomes-based nanoparticles/OVA were fixed at 100 $\mu \mathrm{g} / \mathrm{mL}$ (phytantriol content) and OVA $50 \mu \mathrm{g} / \mathrm{mL}$, while that of GLP was $10 \mu \mathrm{g} / \mathrm{mL}$. Freund adjuvant (FCA) was used as a positive control. Immunization were processed twice at 2-week intervals. Blood were collected 14 days following the second immunization, and serum was collected for OVA-IgG, IgG subtype, and cytokines analysis. Spleens were collected 14 days following the second immunization for cellular immune analysis and RNA-seq.

\section{Analysis of DCs from Lymph Nodes}

The activation of DCs in draining lymph nodes was measured using a previously described method. ${ }^{25,26}$ After injection with OVA or cubosome-based nanoparticles/ OVA at 24 and 48 hours, draining lymph nodes were collected and transferred into $1.5 \mathrm{~mL}$ Eppendorf tubes containing Collagenase D (Roche Applied Science) for preparation of cell suspensions. Then, cell suspensions were filtered with a $40 \mu \mathrm{m}$ Cell Strainer (BD Biosciences) to obtain a single cell suspension. The collected cells were washed and stained with antibodies against CD11c, MHC- II , CD80, and CD86 for 30 minutes at $4{ }^{\circ} \mathrm{C}$. Then, redundant antibodies were removed by washing twice and cells were analyzed using a flow cytometer.

\section{T Cell Proliferation Assay}

Splenocytes from mice were harvested ${ }^{25}$ and cells were washed twice with RPMI 1640 medium containing 10\% FBS and then incubated with OVA at $50 \mu \mathrm{g} / \mathrm{mL}$ for 68 hours. The MTT method was performed for proliferation assay. 


\section{Differentiation of CD4+ and CD8+ T Cells and Cytokines Analysis}

Splenocytes were treated as described above. After incubation with OVA for 68 hours, resulting cells were washed and stained with antibodies against CD3, CD4, and CD8 for 30 minutes at $4^{\circ} \mathrm{C}$ and supernatant were collected for cytokines analysis. Then, cells were washed and analyzed by Flow cytometry instrument.

\section{OVA-IgG, IgG Subtype, and Cytokine}

\section{Analysis}

The detection of OVA-IgG and IgG subtype were performed as previously described. ${ }^{27}$ The suspension of splenocytes medium and serum collected from mice were diluted and analyzed with cytokines ELISA kits (R\&D, Co., USA).

\section{RNA-Seq Analysis in Spleen}

Gene expression levels were measured in spleen by RNAseq sequencing. Spleen from mice were harvested and the total RNA were extracted. A total amount of $2 \mu \mathrm{g}$ RNA per sample was used as input material for the RNA sample preparations. Sequencing libraries were generated using NEBNext UltraTM RNA Library Prep Kit for Illumina (NEB, USA) following manufacturer's recommendations, and index codes were added to attribute sequences to each sample.

\section{Clustering and Sequencing}

Clustering of the index-coded samples was performed on a cBot Cluster Generation System using HiSeq 3000/4000 PE Cluster Kit Box1 (Illumina) according to the manufacturer's instructions. After cluster generation, the library preparations were sequenced on an Illumina Hiseq 4000 platform and $150 \mathrm{bp}$ paired-end reads were generated.

\section{Statistical Analysis}

The experimental data were plotted by GraphPad Prism 6 . Statistical significance was calculated by the one-way ANOVA. All results are presented as mean \pm SEM.

\section{Results and Discussion}

\section{Characterization of GLP and GLPC}

\section{Nanoparticles}

The carbohydrate content of GLP was $99.0 \%$, as determined by the phenol-sulfuric acid method. According to
GPC analysis, the average molecular weight of GLP was $408 \mathrm{Da}$, a single symmetrical peak was also detected by GPC analysis (Figure 1A). As shown in Figure 1B, GLP was composed of Glc, Gal, and Ara in a molar ratio of 4.87:1.99:1.07. The results of GPC and HPLC chromatogram analysis indicated that GLP is an homogeneous oligosaccharide.

The changes in the cubosomes after surface engineering and adsorption of OVA were assessed using a Zetasizer Nano ZS instrument. After surface engineering, the zeta potential of nanoparticles often changes as an indicator of successful modification. Following the addition of CTAB, the zeta potential of CTAB-GLPC increased from -16.97 \pm 1 to $29.17 \pm 0.24 \mathrm{mV}$ (Figure $2 \mathrm{~A}$ ). This indicated that the zeta potential happened inversion, which enabled OVA adsorption. The slow addition of OVA to CTAB-GLPC was performed for the preparation of CTAB-GLPC-OVA, and the zeta potential was measured. As shown in Figure 2A, the zeta potential of CTAB-GLPC-OVA decreased from $29.17 \pm 0.24$ to $19.77 \pm 0.24 \mathrm{mV}$, which was considered to be caused by the adsorption of negatively charged OVA on the CTAB-GLPC surface. The effective size decreased from $324.4 \pm 2.1$ to $303.1 \pm 0.5 \mathrm{~nm}$ after CTAB modification (Figure 2B).

To link PDDAC to the surface of GLPC, PSS was used as transition. Following the linking of PSS on the surface of CTAB-GLPC, the zeta potential of CTABGLPC-PSS was negative $(-30.97 \pm 0.33 \mathrm{mV})$, which created the conditions for PDDAC modification. Then, PDDAC was modified on the surface of CTAB-GLPCPSS and zeta potential of PDDAC-GLPC increased from $-30.97 \pm 0.33$ to $22.97 \pm 0.23 \mathrm{mV}$ (Figure $2 \mathrm{~A}$ ). After OVA adsorption, zeta potential of PDDACGLPC-OVA was $22.13 \pm 0.03 \mathrm{mV}$ and showed no obvious change, which attributed to larger surface area of PDDAC-GLPC likely. PDI is necessary to study the stability of the nanoparticles. As shown in Figure 2C, PDI of CTAB-GLPC was lower compared to other cubosomes-based nanoparticles. The results indicated Surface-engineering has no effect on the stability of the nanoparticles (Figure 2C). The adsorption efficiency of OVA on CTAB-GLPC and PDDAC-GLPC was investigated using a $\mathrm{BCA}$ kit and found to be $52.3 \%$ and $80.9 \%$, respectively.

Phytantriol is known to form a double-diamond cubic phase (Pn3m space group) in excess water or when dispersed as nanoparticles stabilized by pluronic F127. The double-diamond cubic phase (Pn3m) was formed when 

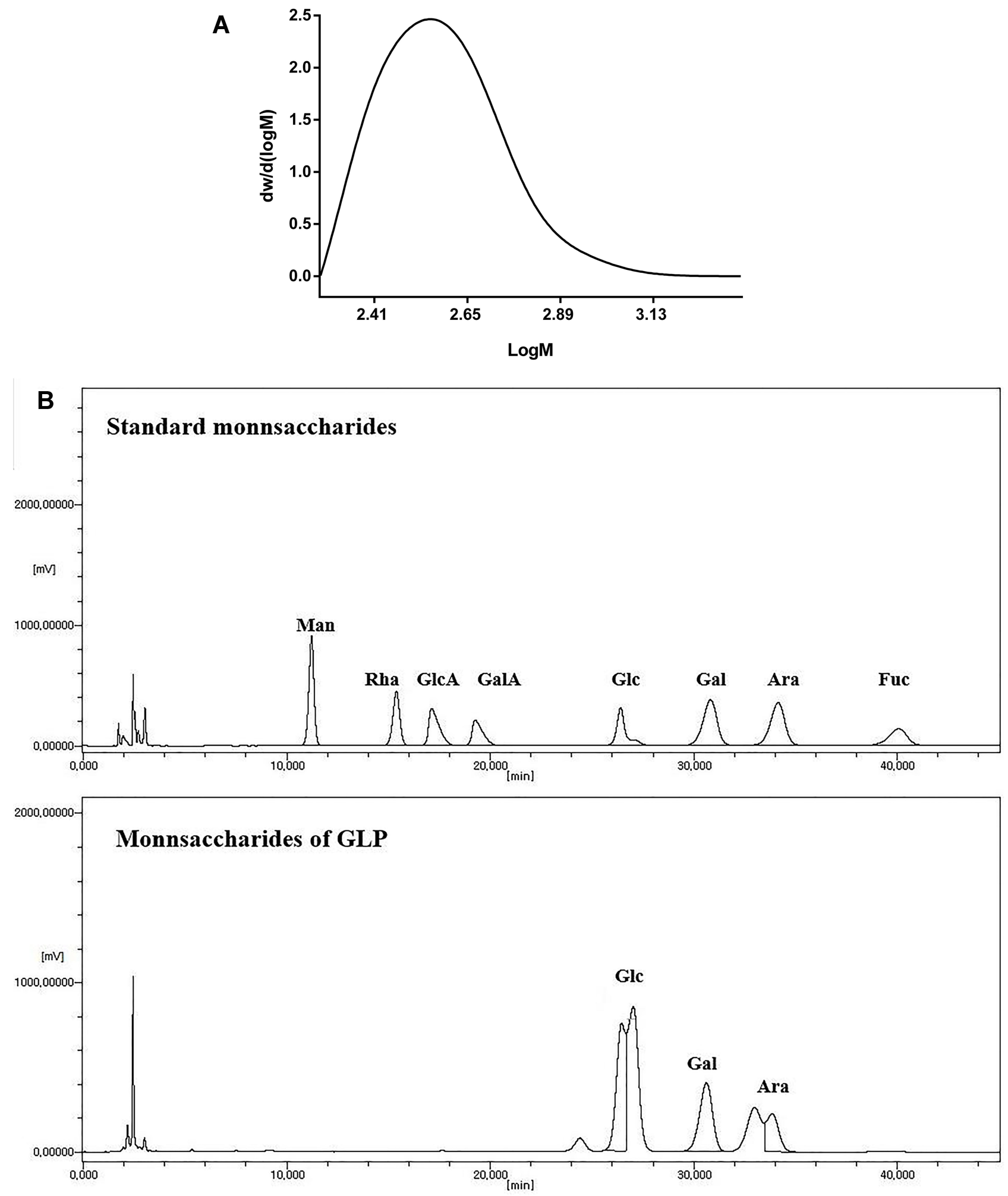

Figure I Characterization of GLP. (A) Molecular weight of GLP was determined using GPC. (B) HPLC chromatogram of hydrolysate from GLP.

phytantriol and pluronic F127 were dispersed in excess water. Our previous studies have indicated that GLPC shows a Pn $3 \mathrm{~m}$ internal structure. ${ }^{28}$ To determine whether surface engineering affects the internal structure of GLPC, SAXS was performed. The SAXS curves were collected at $25^{\circ} \mathrm{C}$ for 30 minutes. As shown in Figure 3, although the 

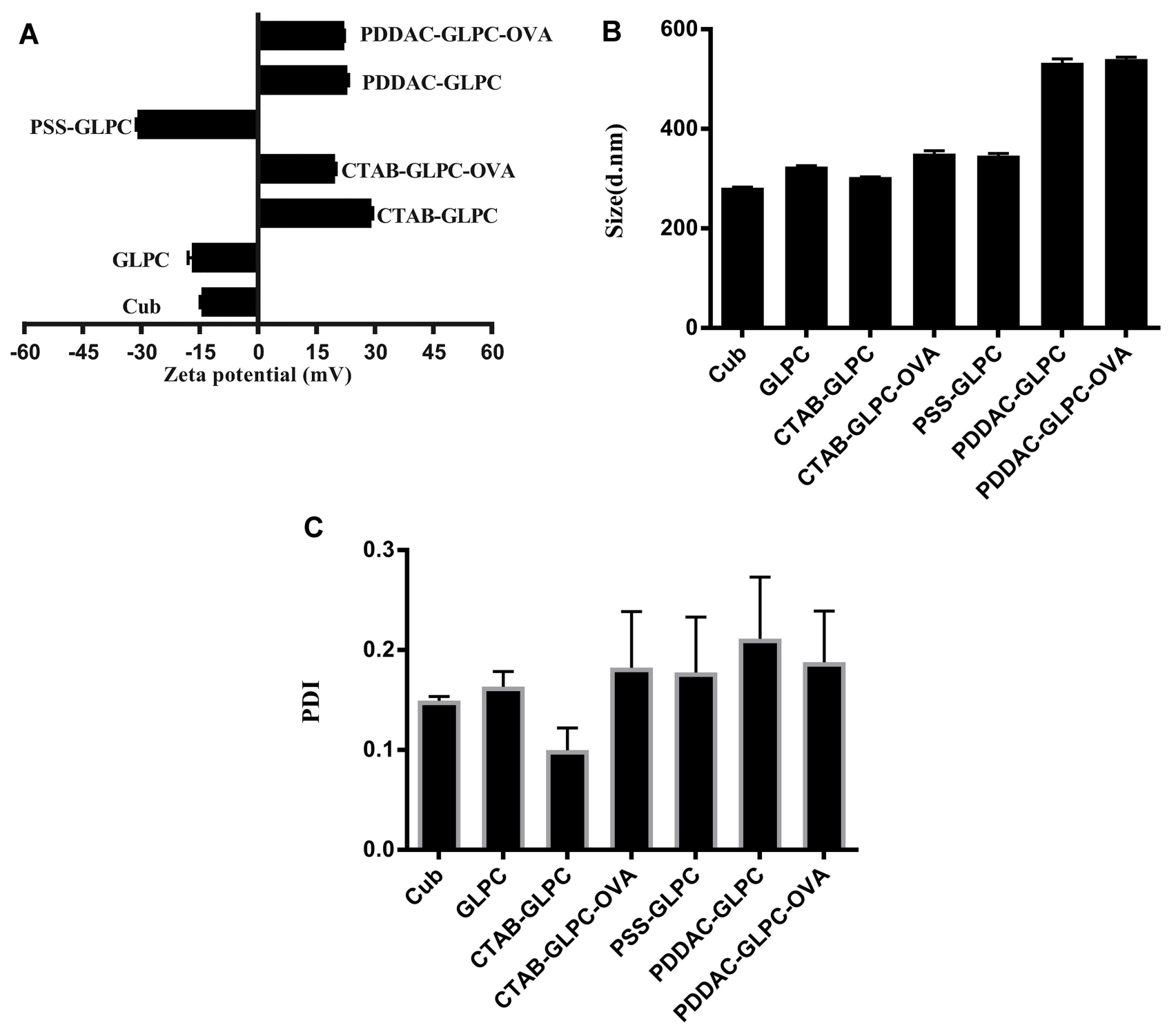

Figure 2 Characterization of Cubosomes-based Nanoparticles. (A) Zeta potential, (B) Size. (C) PDI.

peaks of PDDAC-GLPC and PDDAC-GLPC-OVA moved slightly to the right, three diffraction peaks (relative positions, $\sqrt{2}: \sqrt{ } 3: \sqrt{ } 4)$ were observed, which indicated a Pn3m nanostructure. ${ }^{28}$ The Pn $3 \mathrm{~m}$ nanostructure was retained upon Surface-Engineering and OVA adsorbing. CryoFESEM was performed to investigate the surface structures of cubosome-based nanoparticles. As shown in Figure 4, the nanoparticles showed a cubic morphology and the size of PDDAC-GLPC and PDDAC-GLPC-OVA increased. Cryo-FESEM provides valuable insights into the morphological features of bicontinuous cubic liquid crystalline systems. Some spheres may be formed by freezing water.

\section{Analysis of DCs Maturation}

DCs, which are APCs, play a pivotal role in triggering, enhancing, and linking humoral and cellular immunity. ${ }^{29-34}$ GLPC with different surface coatings (CTAB and PDDAC) were examined to determine whether they could promote the maturation of DCs. Co-stimulatory molecules, such as B7 molecules (CD40 and CD86), play a critical role in regulating T-cell activation and differentiation. ${ }^{34-37}$ Since vaccine was injected and APC (macrophages, DCs, etc.) will be enriched, cubosomes-based nanoparticles would interact with DCs. ${ }^{37-40}$ To test this interaction, DCs were incubated with CTABGLPC-OVA and PDDAC-GLPC-OVA, followed by antibody (CD11c) staining for DCs screening or antibody (CD40 and 

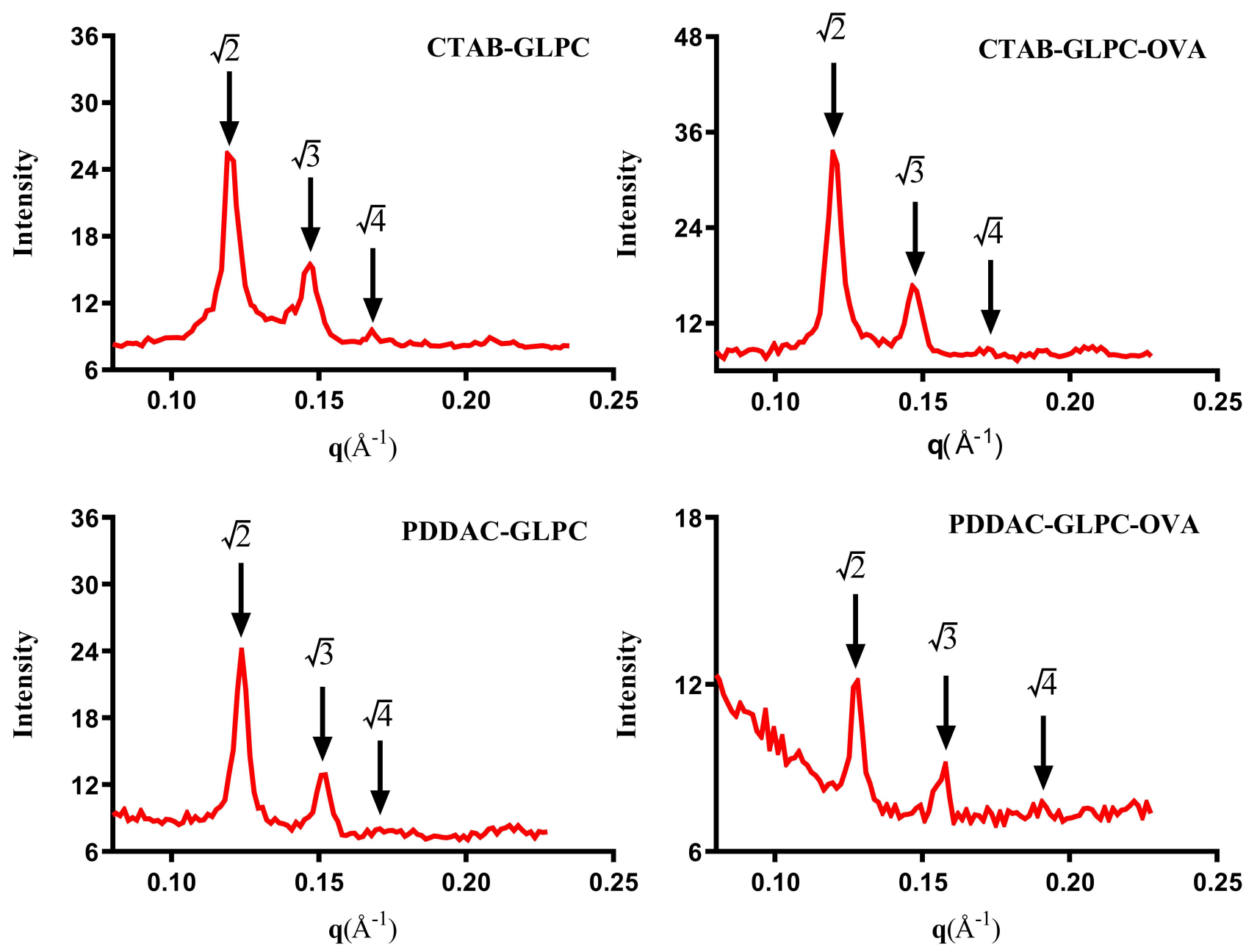

Figure 3 SAXS patterns of CTAB-GLPC, CTAB-GLPC-OVA, PDDAC-GLPC, and PDDAC-GLPC-OVA.

CD86) staining for the upregulation of surface markers to measure DCs maturation. DCs accounts for $90 \%$ of the proportion of the total cell population based on CD11c fluorescence intensity. We found that the percentage of CD40 and CD86 was significantly enhanced $(P<0.01)$ when DCs were treated with PDDAC-GLPC-OVA compared with that when they were treated with GLPC (Figure 5 and Supplementary Figure S1). CD40 and CD86 were up-regulated $(P<0.05)$ when DCs were treated with CTAB-GLPC-OVA relative to GLPC. These results indicate that PDDAC-GLPC-OVA enhanced DC maturation more effectively.

\section{PDDAC-GLPC-OVA Induced Antigen-}

\section{Specific Immunity}

GLPC with different surface coatings (CTAB and PDDAC) were examined to determine whether they could induce antigen-specific immunity after OVA adsorption. When the PDDAC-GLPC-OVA groups were immunized by subcutaneous injection, the results for specific antibodies that are typically representative of humoral immunity showed that the OVA-IgG level was enhanced significantly $(P<0.05)$ compared with that in the GLPC group (Figure 6). However, the CTAB-GLPC-OVA groups did not show stronger immune responses than the GLPC group.

The rapid induction of humoral and cellular immune responses mounts a defensive response against infection. The proliferative ability of splenic lymphocytes is an indirect indicator of an organism's immune status. ${ }^{41-43}$ As shown in Figure 7A, the proliferation index revealed that the PDDACGLPC-OVA and CTAB-GLPC-OVA groups had superior lymphocyte proliferation indices than the GLPC group (Figure 7A). Furthermore, T cell differentiation was examined, and the results showed that CTAB-GLPC-OVA and 

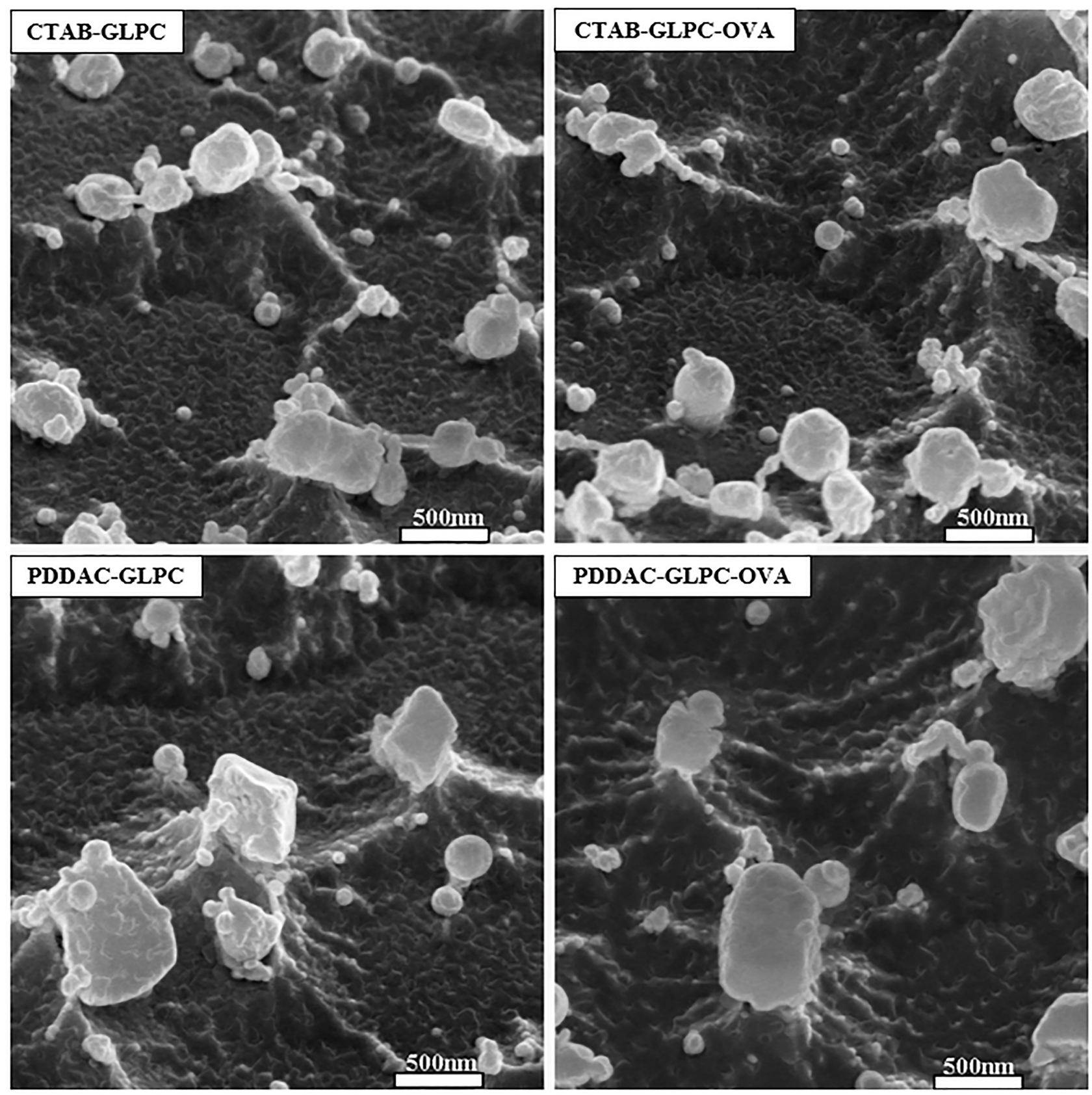

Figure 4 Cryo-FESEM micrographs of CTAB-GLPC, CTAB-GLPC-OVA, PDDAC-GLPC, and PDDAC-GLPC-OVA.

PDDAC-GLPC-OVA have no effect $(P>0.05)$ on the ratio of $\mathrm{CD} 4+/ \mathrm{CD} 8+$ compared with that in the GLPC group (Figure 7B and Supplementary Figure S2). However, after immunization with PDDAC-GLPC-OVA or CTAB-GLPC-OVA, the $\mathrm{T}$ helper cells $(\mathrm{CD} 3+\mathrm{CD} 4+)$ could increase timely and further caused B-cell activation. In other words, after immunization with PDDAC-GLPC-OVA, CD3+ CD8+ T-cells showed rapid proliferation and secretion of various cytokines involved in immune function (Figure $7 \mathrm{C}$ and D). The supernatants of splenic lymphocytes were collected after OVA treatment at 68 hours. IFN- $\gamma$ secretion level was determined by ELISA. When the CTAB-GLPC-OVA and PDDACGLPC-OVA groups were immunized, the IFN- $\gamma$ level was clearly enhanced $(P<0.05)$ compared with that in the single GLPC group (Figure 7E). The result indicates that PDDACGLPC-OVA can generate a rapid and powerful humoral and cellular immune response compared with naked cubosomebased nanoparticles.

To definite the tendency of the immune response, $\operatorname{IgG} 2 \mathrm{a} / \mathrm{IgG} 21$ ratio was used as the indicator of Th1/ 


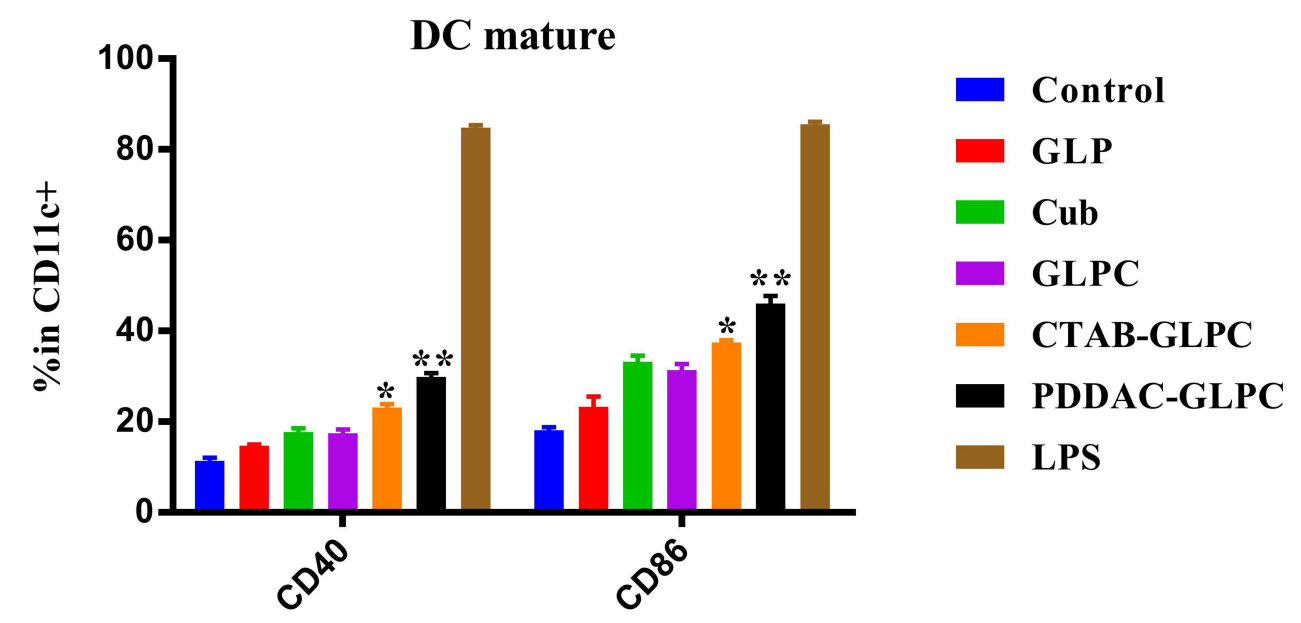

Figure 5 The effects of cubosomes-based nanoparticles on dendritic cell maturation. Data are expressed as the mean \pm SEM. $* P<0.05$ and $* * P<0.01$ vs the GLPC group.

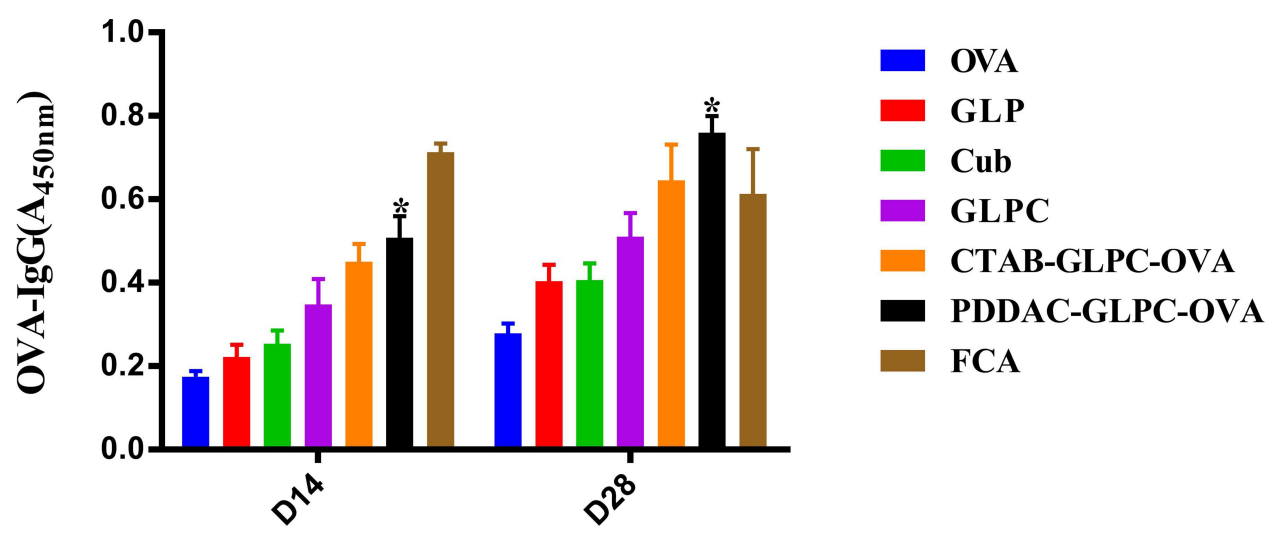

Figure 6 The OVA specific antibody level was measured after final immunization. Data are expressed as the mean \pm SEM. $* P<0.05$ vs the GLPC group.

Th2. ${ }^{44}$ Th1 cells could induce cellular immunity to target cells and clear intracellular pathogens (virus or bacterium), while Th2 cells induce humoral immune response to clear extracellular pathogens. IgG2a/IgG21 in the cubosomesbased nanoparticles increased significantly compared to the naked OVA, which indicates a Th2 immune response. However, the value of $\operatorname{IgG} 2 \mathrm{a} / \operatorname{IgG} 21$ has no significant $(P>0.05)$ difference among GLPC, CTAB-GLPC-OVA, and PDDAC-GLPC-OVA group (Figure 7F). The result indicated the tendency of the immune response had not been changed by Surface-Engineering.

\section{DCs Maturation in Draining Lymph Nodes}

The results showed that cubosomes-based nanoparticles with different modification showed different adjuvant activity effects in vivo. The mechanism underlying the action of PDDAC-GLPC-OVA as an effective adjuvant and the effect of modifications on triggering the immune response were explored. As the initial site at which the immune response to a vaccine occurs, draining lymph nodes play an important role in triggering the immune response. After the uptake of vaccine antigens at the injection site, DCs migrate to nearby draining lymph nodes and mature during the process of migration. Then, mature DCs interact with $\mathrm{T}$ and B lymphocytes via their surface molecules. We next investigated how surface engineering affects adjuvant activity in terms of activating DCs in the draining lymph nodes. The activation markers (CD80, CD86, and MHC II) of DCs were examined in the lymph node after primary immunization at 24 hours and 48 hours. The expression of CD80, CD86, and MHC II in CTAB-GLPC-OVA and PDDACGLPC-OVA groups was higher than in the naked OVA group $(P<0.05)$. At 24 hours, only the expression of CD80 in the CTAB-GLPC-OVA and PDDAC-GLPC-OVA groups was higher than in the GLPC group (Figure 8A and Supplementary Figure S3). However, CD80 and 

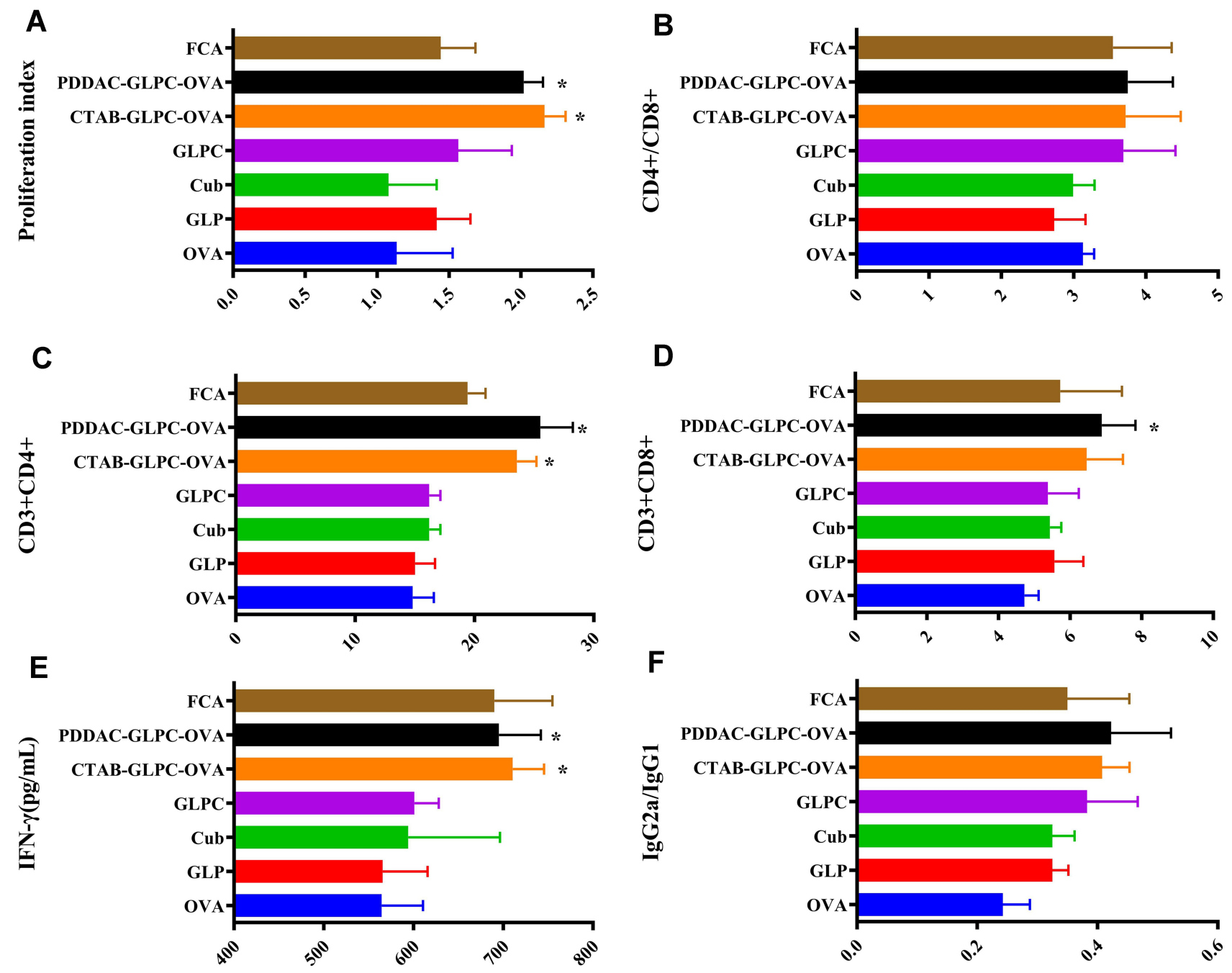

Figure 7 The effects of cubosome-based nanoparticles on splenic T-cells after OVA re-stimulation, and the level of lgG2a/lgG in serum. (A) Spleen lymphocyte proliferation index after OVA re-stimulation. (B) CD4+ to CD8+ T-cell ratio. (C) CD3+CD4+ T-cell proliferation. (D) CD3+CD8+ T-cell proliferation. (E) IFN- $\gamma$ secretion by splenocytes after OVA re-stimulation. (F) lgG2a to lgGI ratio in serum; data are expressed as the mean $\pm \mathrm{SEM}$; $* P<0.05$ vs. the $G L P C$ group.

CD86 were significantly more highly expressed in mice injected with CTAB-GLPC-OVA and PDDAC-GLPCOVA than in those injected with naked GLPC at 48 hours (Figure 8B and Supplementary Figure S4) $(P<0.05)$, which indicates that CTAB-GLPC-OVA and PDDAC-GLPCOVA can induce the activation and maturation of DCs more effectively than naked cubosome-based nanoparticles in vivo.

\section{Cytokine Levels in Serum}

The change of serum cytokines has an important value to assess the activity of adjuvant in systemic circulation. ${ }^{45}$ To measure the immune response in systemic circulation, the levels of IFN- $\gamma$, TNF- $\alpha$, IL-4, and IL-6 in serum were measured by ELISA at D14 after final vaccination. As shown in Figure $8 \mathrm{C}$ and $\mathrm{D}$, mice injected with PDDAC-
GLPC-OVA generated higher production of IFN- $\gamma$, TNF- $\alpha$, and IL-4 compared to GLPC. However, mice injected with CTAB-GLPC-OVA only generated higher $(P<0.05)$ production of IL-4. These results suggested that PDDACGLPC-OVA could significantly enhance both the Th1 and Th2 immune response. Overall, serum cytokines were enhanced after Surface-Engineering in systemic circulation, which suggested PDDAC modification can effectively cause a systemic immune response.

\section{General Genetic Expression of the Spleen}

Immune function is the most important function of the spleen, which contains a variety of immune cells that interact with immune factors. ${ }^{46}$ The spleen plays specific roles in the immune response through cell-mediated cellular and humoral immunity. ${ }^{47}$ The function of the spleen 

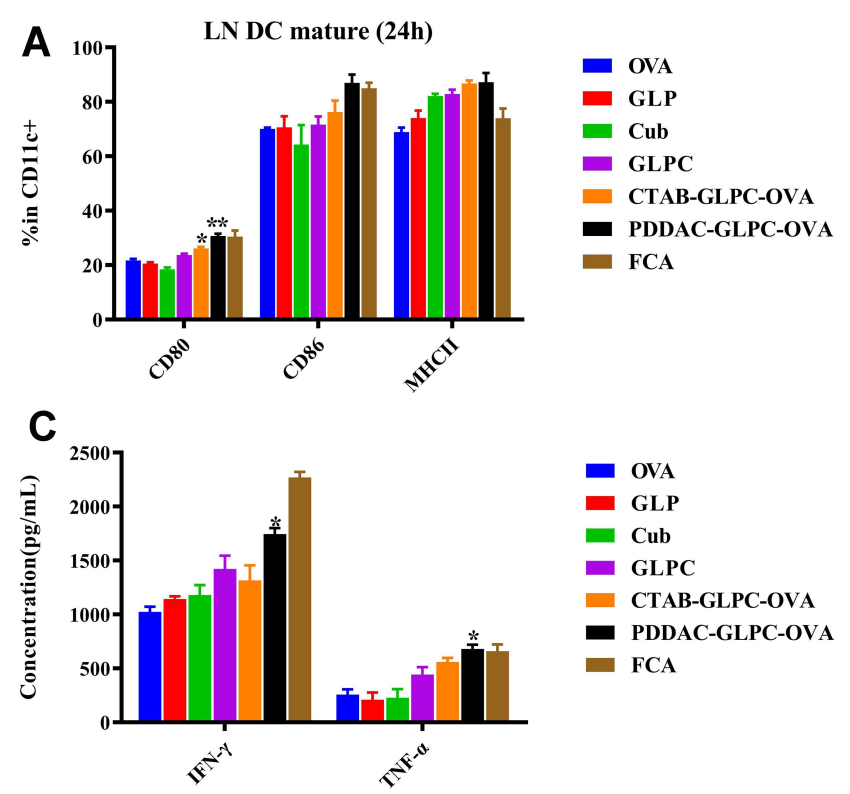
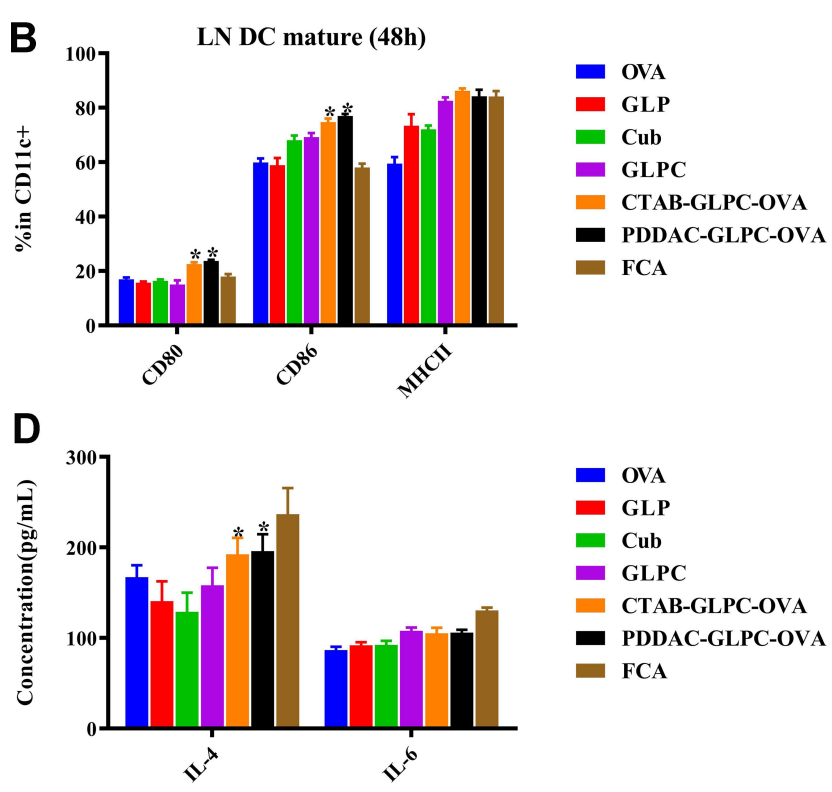

Figure 8 Activating DCs in the draining lymph nodes at 24 hours (A) and 48 hours (B) after the first immunization. Production of IFN- $\gamma$ and TNF- $\alpha$ in the serum of mice (C), IL-4, and IL-6 (D) in the serum of mice. Data are expressed as the mean \pm SEM. $* P<0.05$ and $* * P<0.0$ I vs the GLPC group.

is indirectly influenced by draining lymph nodes. Then, the function of the spleen interacted with systemic circulation. We have found PDDAC-GLPC-OVA can induced promoted the more effective promotion of the activation of DCs compared to more effectively than did naked cubosomes-based nanoparticles in vivo; further, they promoted the production of OVA-IgG in the serum. How they affected draining lymph nodes and further affected systemic circulation. Spleens were collected at day 14 after final immunization, and the total RNA extracted from the OVA, GLPC, and PDDAC-GLPC-OVA group was analyzed using RNA-seq.

As shown in Figure 9A, 368 differentially expressed genes were identified, including 292 up-regulated and 76 down-regulated genes $(P<0.05)$ in the PDDAC-GLPCOVA group relative to the GLPC group (Figure 9A). Hierarchical clustering showed that the mechanism of action underlying the effect of PDDAC-GLPC-OVA on the spleen was significantly different from that of GLPC or OVA (Figure 9B). The results indicate the mechanism of action of cubosome-based nanoparticles changes after PDDAC modification.

The immune-related signaling pathways were screened by KEGG pathway analysis. As shown in Figure 9C, the adjuvant activity of the PDDAC-GLPC-OVA in the spleen was induced by the activation of leukocyte transendothelial migration, cytokine-cytokine receptor interaction, and complement and coagulation cascades. We were not sure if these cytokines identified in this study were OVA specific, but we believe they did regulate the immune cell homing in response to antigen. The leukocyte transendothelial migration pathway was also found in the KEGG enrichment in this study, which confirmed that intensive immune cell migration had occurred in immune response. Further study will be needed to identify the exact role of the cytokine-cytokine receptor interaction and leukocyte transendothelial migration identified in this study, and to identify what immune cells are involved and how they are attracted to the immune response after vaccine.

\section{Conclusion}

In this study, we investigated the adjuvant activity of surface-engineered GLP cubosomes, and demonstrated that the adjuvant activity of GLPC was influenced by surface engineering. For cubosome-based nanoparticles, CTABGLPC-OVA and PDDAC-GLPC-OVA showed a stable state and retained their $\mathrm{Pn} 3 \mathrm{~m}$ nanostructure. Because of the PDDAC modification, the compound had excellent features and promoted DC maturation and antigen uptake. The results showed that CTAB-GLPC-OVA failed to promote the humoral immune response relative to GLPC and PDDAC-GLPC-OVA, which were both found to activate and promote humoral and cellular immune responses. PDDAC-GLPC-OVA strongly promoted the proliferation of $\mathrm{CD} 3+\mathrm{CD} 4+$ or $\mathrm{CD} 3+\mathrm{CD} 8+\mathrm{T}$-cells; furthermore, it effectively modulated the expression of some cytokines, 


\section{A PDDAC-GLPC-OVA VS GLPC}

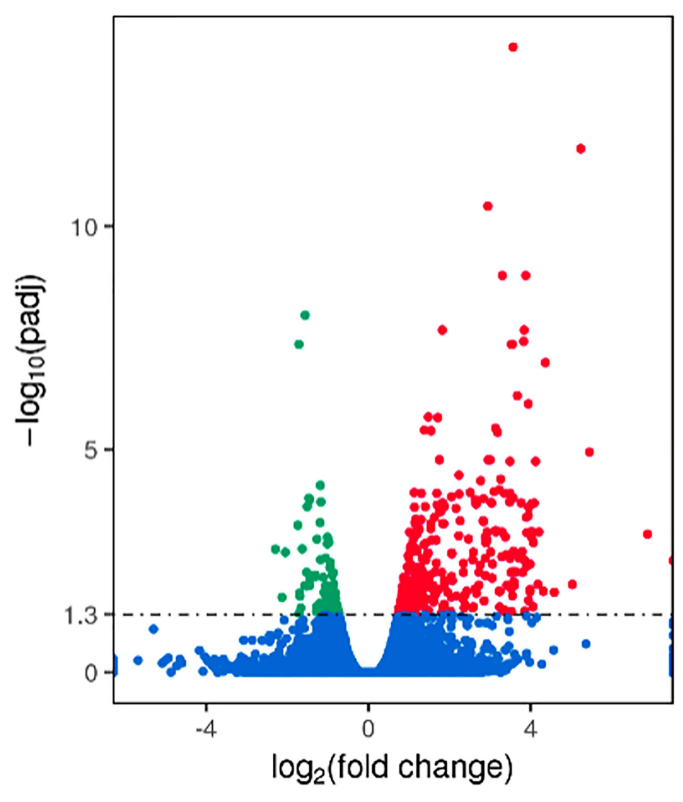

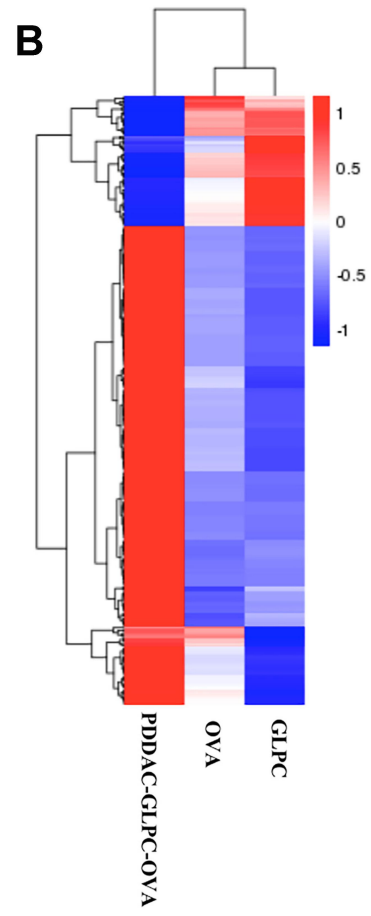

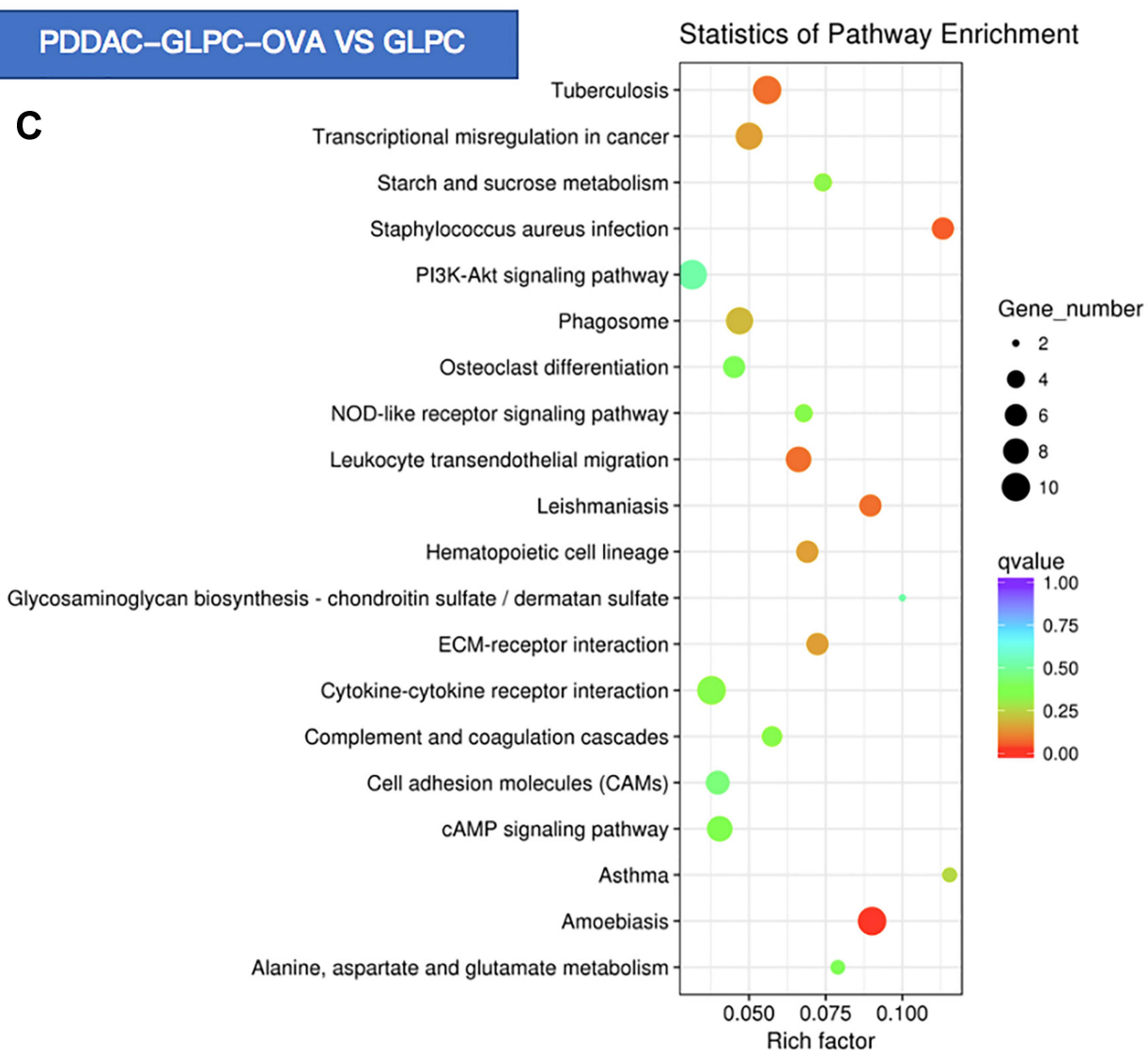

Figure 9 (A) Expression profiles of mRNAs in the spleen. (B) Hierarchical clustering of genes differentially expressed in PDDAC-GLPC-OVA vs OVA vs GLPC. (C) KEGG pathway analysis in the PDDAC-GLPC-OVA group vs the GLPC group.

Differential Expressed Genes ( 368 )

- up regulated: 292

- down regulated: 76
Gene number

- 2

4

6
8
10

qvalue

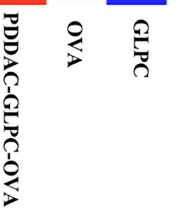

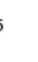


including IFN- $\gamma$, TNF- $\alpha$, IL-4, and IL- 6 . The enhanced immune responses of PDDAC-GLPC-OVA might be attributed to the maturation of DCs into draining lymph nodes, activation of the spleen, and secretion of cytokines into systemic circulation. The current work indicates that PDDAC modification is beneficial for enhancing the humoral and cellular immune response, suggesting that PDDAC-GLPC-OVA is a potential adjuvant for proteinantigen vaccines.

\section{Abbreviations}

GLP, Ganoderma lucidum polysaccharide; GLPC, Ganoderma lucidum polysaccharide cubosomes; CTAB, cetyltrimethylammonium bromide; PDDAC, poly (diallydimethy ammoniumchloride); PSS, poly(styrenesulfate); GPC, gel permeation chromatography; HPLC, High Performance Liquid Chromatography; MTT, 3-(4,5-dimethylthiazol-2-yl)-2,5-diphenyltetrazolium bromide; PDI, polydispersity index; SAXS, X-ray scattering; Cryo-FESEM, cryo-field emission scanning electron microscopy; DCs, dendritic cells.

\section{Acknowledgments}

We are grateful to all the staff in the Institute of Traditional Chinese Veterinary Medicine of Nanjing Agricultural University for their assistance with experiments.

\section{Funding}

The project was supported by the National Natural Science Foundation of China (Grant No. 31672596), Natural Science Foundation of Jiangsu Province (Grant No. BK20190521) and a Project Funded by the Priority Academic Program Development of Jiangsu Higher Education Institutions (PAPD).

\section{Disclosure}

The authors report no conflicts of interest for this work.

\section{References}

1. Gupta RK, Rost BE. Aluminum compounds as vaccine adjuvants. $A d v$ Drug Deliv Rev. 1998;32(3):155-172. doi:10.1016/S0169-409X(98) 00008-8

2. Stephenson I, Bugarini R, Nicholson KG, et al. Cross-reactivity to highly pathogenic avian influenza $\mathrm{H} 5 \mathrm{~N} 1$ viruses after vaccination with nonadjuvanted and MF59-adjuvanted influenza a/duck/Singapore/97 (H5N3) vaccine: a potential priming strategy. J Inf Dis. 2005;191 (8):1210-1215. doi:10.1086/428948

3. Nohynek H, Jokinen J, Partinen M, et al. AS03 adjuvanted AH1N1 vaccine associated with an abrupt increase in the incidence of childhood narcolepsy in Finland. PLoS One. 2012;7(3):e33536.
4. Ouyang XH, Ouyang Y. Nanomaterials and nanotechnology as well as its application in medicine: a comprehensive review with graphics. J Interventional Radiol. 2012;21(7):529-535.

5. Zhang L, Webster TJ. Nanotechnology and nanomaterials: promises for improved tissue regeneration. Nano Today. 2009;4(1):66-80. doi:10.1016/j.nantod.2008.10.014

6. Christensen D, Korsholm KS, Rosenkrands I, Lindenstrom T, Andersen P, Agger EM. Cationic liposomes as vaccine adjuvants. Expert Rev Vaccines. 2011;10(4):513-521. doi:10.1586/erv.11.17

7. Sivakumar SM, Sukumaran N, Murugesan R, et al. Immune augmentation of single contact hepatitis B vaccine by using PLGA microspheres as an adjuvant. Indian J Pharm Sci. 2008;70(4):487-490. doi:10.4103/0250-474X.44599

8. Rizwan SB. Cubosomes as Novel Antigen Delivery Systems. University of Otago School of Pharmacy. 2009.

9. Nielsen LH, Rades T, Boyd B, Boisen A. Microcontainers as an oral delivery system for spray dried cubosomes containing ovalbumin. Eur J Pharm Biopharm. 2017;118:13-20. doi:10.1016/j.ejpb.2016.12.008

10. Rizwan SB, Mcburney WT, Young K, et al. Cubosomes containing the adjuvants imiquimod and monophosphoryl lipid A stimulate robust cellular and humoral immune responses. $J$ Control Release. 2013;165(1):16-21. doi:10.1016/j.jconrel.2012.10.020

11. Kojarunchitt T, Baldursdottir S, Dong YD, Boyd BJ, Rades T, Hook S. Modified thermoresponsive poloxamer 407 and chitosan sol-gels as potential sustained-release vaccine delivery systems. Eur J Pharm Biopharm. 2015;89:74-81. doi:10.1016/j.ejpb.2014.11.026

12. Xing-Feng B, Xue-Song W, Qun D, Ji-Nian F, Xiao-Yu L. Structural features of immunologically active polysaccharides from ganoderma lucidum. Ganoderma Lucidum Phytochem. 2002;59(2):175-181. doi:10.1016/S0031-9422(01)00450-2

13. Kuo-I L, Yeong-Yi K, Hui-Kai K, et al. Reishi polysaccharides induce immunoglobulin production through the TLR4/ TLR2-mediated induction of transcription factor Blimp-1. J Biol Chem. 2006;281(34):24111-24123. doi:10.1074/jbc.M601106200

14. Angelov B, Angelova A, Papahadjopoulos-Sternberg B, Hoffmann SV, Nicolas V, Lesieur S. Protein-containing PEGylated cubosomic particles: freeze-fracture electron microscopy and Synchrotron Radiation Circular Dichroism Study. J Phys Chem B. 2012;116(26):7676-7686.

15. Angelova A, Angelov B, Papahadjopoulos-Sternberg B, et al. Proteocubosomes: nanoporous vehicles with tertiary organized fluid interfaces. Langmuir. 2005;21(9):4138-4143. doi:10.1021/la047745t

16. Rizwan SB, Assmus D, Boehnke A, et al. Preparation of phytantriol cubosomes by solvent precursor dilution for the delivery of protein vaccines. Eur J Pharm Biopharm. 2011;79(1):15-22. doi:10.1016/j. ejpb.2010.12.034

17. Zhai J, Fong C, Tran N, et al. Non-lamellar lyotropic liquid crystalline lipid nanoparticles for the next generation of nanomedicine. ACS Nano. 2019;13(6):6178-6206. doi:10.1021/acsnano.8b07961

18. Mulet X, Boyd BJ, Drummond CJ. Advances in drug delivery and medical imaging using colloidal lyotropic liquid crystalline dispersions. J Colloid Interface. 2013;393:1-20. doi:10.1016/j.jcis.2012.10.014

19. Jian X, Ligeng X, Hua G, et al. Antigen-loaded upconversion nanoparticles for dendritic cell stimulation, tracking, and vaccination in dendritic cell-based immunotherapy. Acs Nano. 2015;9(6):6401-6411.

20. Sun B, Ji Z, Liao YP, et al. Engineering an effective immune adjuvant by designed control of shape and crystallinity of aluminum oxyhydroxide nanoparticles. Acs Nano. 2013;7(12):10834-10849. doi: $10.1021 / \mathrm{nn} 404211 \mathrm{j}$

21. Deshpande S, Venugopal E, Ramagiri S, Bellare JR, Kumaraswamy G, Singh N. Enhancing cubosome functionality by coating with a single

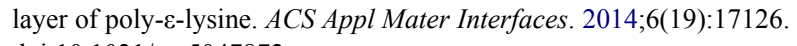
doi:10.1021/am5047872

22. Lynn GM, Richard L, Darrah PA, et al. In vivo characterization of the physicochemical properties of polymer-linked TLR agonists that enhance vaccine immunogenicity. Nat Biotechnol. 2015;33 (11):1201-1210. doi:10.1038/nbt.3371 
23. Xu L, Liu Y, Chen Z, et al. Surface-engineered gold nanorods: promising DNA vaccine adjuvant for HIV-1 treatment. Nano Lett. 2012;12(4):2003-2012. doi:10.1021/nl300027p

24. Zhao L, Seth A, Wibowo N, et al. Nanoparticle vaccines. Vaccine. 2014;32(3):327-337. doi:10.1016/j.vaccine.2013.11.069

25. Liu Z, Luo L, Zheng S, et al. Cubosome nanoparticles potentiate immune properties of immunostimulants. Int $J$ Nanomedicine. 2016;11:3571-3583.

26. Lutz MB, Kukutsch N, Ogilvie ALJ, et al. An advanced culture method for generating large quantities of highly pure dendritic cells from mouse bone marrow. J Immunol Methods. 1999;223(1):77-92. doi:10.1016/S0022-1759(98)00204-X

27. Liu Z, Xing J, Zheng S, et al. Ganoderma lucidum polysaccharides encapsulated in liposome as an adjuvant to promote Th1-bias immune response. Carbohydr Polym. 2016;142:141-148. doi:10.1016/j. carbpol.2016.01.021

28. Fraser S. Cubic Phase Lipids as Novel Biosensing Surfaces. School of Chemistry; 2011.

29. Uematsu S, Fujimoto K, Yang MB, et al. Regulation of humoral and cellular gut immunity by lamina propria dendritic cells expressing toll-like receptor 5. Nat Immunol. 2008;9(7):769-776. doi:10.1038/ ni. 1622

30. Allavena P, Bottazzi B, Doni A, Romani L, Garlanda C, Mantovani A, Production of the long pentraxin PTX3 by myeloid dendritic cells: linking cellular and humoral innate immunity, 2008.

31. Ueno H, Schmitt N, Palucka AK, Banchereau J. Dendritic cells and humoral immunity in humans. Immunol Cell Biol. 2010;88 (4):376-380. doi:10.1038/icb.2010.28

32. Kikuchi T, Worgall S, Singh R, et al. Dendritic cells genetically modified to express CD40 ligand and pulsed with antigen can initiate antigen-specific humoral immunity independent of CD4+ T cells. Nat Med. 2000;6(10):1154-1159. doi:10.1038/80498

33. Li L, Schmitt A, Reinhardt P, et al. Reconstitution of CD40 and CD80 in dendritic cells generated from blasts of patients with acute myeloid leukemia. Cancer Immun. 2003;3(63):8.

34. Gurunathan S, Irvine $\mathrm{KR}, \mathrm{Wu} \mathrm{CY}$, et al. CD40 ligand/trimer DNA enhances both humoral and cellular immune responses and induces protective immunity to infectious and tumor challenge. J Immunol. 1998;161(9):4563-4571.

35. Mckay PF, Dan H, Sampa BS, et al. Recruitment of different subsets of antigen-presenting cells selectively modulates DNA vaccine-elicited CD4+ and CD8+ T lymphocyte responses. Eur J Immunol. 2004;34 (4):1011-1020.
36. Nakanishi T, Ototake M. Antigen uptake and immune responses after immersion vaccination. Fish Vaccinology. 1997;90(90):59-68.

37. Oh W-K, Kim S, Choi M, et al. Cellular uptake, cytotoxicity, and innate immune response of silica-titania hollow nanoparticles based on size and surface functionality. Acs Nano. 2010;4(9):5301-5313. doi:10.1021/nn100561e

38. Kemnade JO, Seethammagari M, Collinson-Pautz M, Kaur H, Spencer DM, Mccormick AA. Tobacco mosaic virus efficiently targets DC uptake, activation and antigen-specific $\mathrm{T}$ cell responses in vivo. Vaccine. 2014;32(33):4228-4233. doi:10.1016/j.vaccine.2014. 04.051

39. Platzer B, Stout M, Fiebiger E. Antigen cross-presentation of immune complexes. Front Immunol. 2014;5:140. doi:10.3389/fimmu.2014.00 140

40. Bürdek M, Spranger S, Wilde S, Frankenberger B, Schendel DJ, Geiger C. Three-day dendritic cells for vaccine development: antigen uptake, processing and presentation. J Transl Med. 2010;8(1):90.

41. Jing C, Ning Q, Xia YM. Effect of zinc on immune function in mice spleen lymphocytes. Wei Sheng Yan Jiu. 2005;34(6):710.

42. Nossal GJ, Austin CM, Pye J, Mitchell J. Antigens in immunity. XII. Antigen trapping in the spleen. Int Arch Allergy Appl Immunol. 1966;29(4):368-383. doi:10.1159/000229718

43. Kocabas AM, Li P, Cao D, et al. Expression profile of the channel catfish spleen: analysis of genes involved in immune functions. Mar Biotechnol. 2002;4(6):526-536. doi:10.1007/s10126-002-0067-0

44. Huber VC, Mckeon RM, Brackin MN, et al. Distinct contributions of vaccine-induced immunoglobulin G1 (IgG1) and $\mathrm{IgG} 2 \mathrm{a}$ antibodies to protective immunity against influenza. Clin Vaccine Immunology Cvi. 2006;13(9):981-990. doi:10.1128/CVI.00156-06

45. Rendina DN, Ryff CD, Coe CL. Concordance of serum cytokines and stimulated mononuclear cell responses in older adults. Brain Behav Immun. 2015;49:e39-e39. doi:10.1016/j.bbi.2015.06.148

46. Kisseleva T, Köckritz-Blickwede MV, Reichart D, et al. Fibrocytelike cells recruited to the spleen support innate and adaptive immune responses to acute injury or infection. $J$ Mol Med. 2011;89 (10):997-1013. doi:10.1007/s00109-011-0756-0

47. Ellis EF, Smith RT. The role of the spleen in immunity. With special reference to the post-splenectomy problem in infants. Pediatrics. 1966;37(1):111-119.
International Journal of Nanomedicine

\section{Publish your work in this journal}

The International Journal of Nanomedicine is an international, peerreviewed journal focusing on the application of nanotechnology in diagnostics, therapeutics, and drug delivery systems throughout the biomedical field. This journal is indexed on PubMed Central, MedLine, CAS, SciSearch ${ }^{\mathbb{}}$, Current Contents ${ }^{\mathbb{R}} /$ Clinical Medicine, $^{-}$

\section{Dovepress}

Journal Citation Reports/Science Edition, EMBase, Scopus and the Elsevier Bibliographic databases. The manuscript management system is completely online and includes a very quick and fair peer-review system, which is all easy to use. Visit http://www.dovepress.com/ testimonials.php to read real quotes from published authors. 